\title{
Reformas económicas, inversión extranjera directa y cambios en la estructura de la industria automotriz china (1980-2004)
}

\author{
Lourdes Álvarez Medina* \\ Elizabeth Sepúlveda Reyes**
}

\section{Resumen}

En este trabajo se estudian los cambios ocurridos en la industria automotriz china a partir de las reformas económicas de la década de los ochenta, las cuales buscaban transformar una industria automotriz fragmentada, sin recursos financieros y con tecnologías obsoletas, en un oligopolio en el que dominaran grupos automotrices chinos y la inversión extranjera participara con recursos financieros, tecnológicos y conocimientos administrativos. Asimismo, se observa que mediante la adecuada participación del Estado en la industria y en la armonización en el cambio de políticas de inversión extranjera del sector automotriz comerciales y de consumo, se logró un crecimiento extraordinario de la producción y un cambio cercano a lo planeado en la estructura de la industria. La lenta evolución de medidas proteccionistas y apertura comercial permitieron la creación de importantes capacidades de diseño y producción. Existen beneficios a partir de la inversión extranjera como la participación en la creación de capital bruto, la creación de empleo y las aportaciones fiscales. La industria automotriz en China se perfila como una de las más importantes del mundo; para México, especialmente, se tornará en un competidor importante en el mercado de autopartes de los Estados Unidos, por lo que seguir su evolución se vuelve imprescindible.

Palabras clave: China, industria, automotriz, inversión extranjera.

\section{Introducción}

\section{A partir de las reformas económicas iniciadas en los ochenta, ha sido muy Acriticada la intervención del Estado en la economía; sin embargo, el estudio}

\footnotetext{
*Investigadora de la División de Investigación de la Facultad de Contaduría y Administración, UNAM. Correo electrónico: lourdes3055@yahoo.com

**Alumna de la División de Estudios de Posgrado de la Facultad de Contaduría y Administración de la UNAM. Correo electrónico: elizabethsereyes@yahoo.com.mx
} 
de la industria automotriz en China nos permite ver que gracias a esa intervención el capital chino actualmente tiene oportunidades y una participación importante en la industria. El gobierno ha sido el principal responsable del crecimiento y desarrollo asegurando la inversión extranjera directa y la participación del capital chino. Al mismo tiempo ha buscado crear capacidades locales de diseño y producción mediante la regulación y aplicación adecuada de las leyes de inversión extranjera directa y de la política industrial automotriz.

La industria automotriz china surgió en 1953 con la construcción de la First Auto Works (FAW), pero es a partir de la década de los noventa cuando creció extraordinariamente; en 2004 alcanzó una cifra récord de producción de 5 millones de unidades, convirtiéndose en el cuarto productor mundial después de EUA, Japón y Alemania.

China tiene 1300 millones de habitantes — que representan aproximadamente el $20 \%$ de la población mundial- y 827 millones de población empleada. La población urbana pasó del 19.6 al 37.6\% entre 1980 y 2002, el Producto Interno Bruto (PIB) creció a una tasa promedio anual de $8 \%$ en los últimos 22 años y la tasa de inflación y el déficit fiscal son inferiores al 3\% (Dussel, 2003: 7).

Actualmente existen 0.0099 vehículos per cápita, un mercado meta de 200 millones de consumidores y la producción de automóviles creció $276 \%$ en la última década. Se pronostica que la producción alcanzará los diez millones de vehículos por año en 2010, con lo que se ubicaría en el segundo lugar de producción mundial, solamente después de los Estados Unidos (Fourin, 2004a).

En 1980, China comenzó un periodo de reformas económicas y de apertura comercial, lo que promovió el desarrollo de la industria automotriz. Las empresas estatales se convirtieron en sociedades por acciones, vendiéndose el $49 \%$ a socios extranjeros y el 51\% quedó en poder del Estado. El crecimiento de la industria automotriz respondió a una estrategia diseñada por el gobierno en la que se buscó la creación de empresas fabricantes de automóviles con capital mayoritariamente chino, que operasen globalmente y pudiesen competir mundialmente. Así como Renault de Francia y Volkswagen de Alemania, el gobierno planeó la creación de sus grandes grupos automotrices: First Automotive Group (FAW), Shanghai Automotive Group Co. (SAIC), entre otros. 
En la industria automotriz china coexisten dos sistemas de producción de vehículos: los que producen las empresas mundiales, que son los convencionales o clásicos, y los fabricados por empresas de capital local, que son conocidos como vehículos de campo y evolucionan a partir de la tecnología para fabricar motocicletas y tractores (Wang y Richet, 2001: 1).

En este trabajo se considera exclusivamente el sector de vehículos clásicos porque es el que recibe la inversión extranjera directa (IED). Además, se analizan los cambios ocurridos en la industria automotriz china a partir de las reformas económicas de la década de los ochenta. Específicamente nos interesa saber cuáles son y cómo afectan los cambios institucionales al crecimiento y a la estructura de la industria. Se analiza la regulación de la IED, la política de la industria automotriz, el crecimiento y los cambios en la estructura de esta industria. Nuestra investigación se basa en fuentes secundarias: estadísticas proporcionadas por la CEPAL, por la Industria Nacional de Autopartes, despachos internacionales como Coudert Brothers, Fourin y McKinsey, tesis ${ }^{1}$, libros y revistas especializadas.

El artículo está organizado en cinco partes: en la primera se hacen algunas consideraciones teóricas para el análisis; en la segunda se presenta información general de la industria automotriz china, su crecimiento y evolución; en la tercera se discuten cambios institucionales que incentivan o frenan el desarrollo de la industria como la protección arancelaria, la política de consumo, la regulación de la inversión extranjera directa y la política automotriz; en la cuarta se analiza la participación de la inversión extranjera directa y sus resultados con relación a productividad, utilidades, retorno sobre la inversión y ventas; en la quinta se describe la estructura de la industria automotriz, la participación en el mercado de los grupos chinos y de las Joint Ventures (JV); finalmente, se presentan las conclusiones.

\section{Consideraciones teóricas}

Las teorías del desarrollo económico que han tenido una evolución muy controvertida han sido criticadas por haber tendido a hacer generalizaciones inadecuadas en su afán por construir una teoría general para el Tercer Mundo; se considera que fueron demasiado simplistas e ignoraron los aspectos sociales y políticos de la transformación;

\footnotetext{
${ }^{1}$ Se expresa un agradecimiento especial al Dr. Wang por proporcionarnos su tesis doctoral e intercambiar opiniones sobre el futuro de la industria automotriz china.
} 
pusieron excesivo énfasis en agregados y medidas nacionales; no abordaron suficientemente los problemas de distribución espacial, sectorial o interpersonal; no tomaron en cuenta la realidad, como en la teoría de las etapas, en la que se proponía que había que pasar por varias etapas para alcanzar un crecimiento autosostenido; y en ocasiones demasiado pesimistas como la teoría del subdesarrollo (Bustelo, 1999: 13).

En las teorías del desarrollo se identifican dos grandes corrientes: los estructuralistas y los neoclásicos. Los primeros afirman que para el estudio de los países subdesarrollados se necesita un marco teórico y analítico distinto al existente porque las economías de los países pobres son inflexibles; esto quiere decir que el cambio se ve inhibido por las instituciones, cuellos de botella, la oferta de casi todos los bienes y servicios es inelástica, los mercados de productos y factores son a menudo imperfectos y estos problemas estructurales impiden el proceso de desarrollo. Por el contrario, la economía neoclásica parte del supuesto de que las economías de los países son flexibles; se caracterizan por un comportamiento económico racional, con agentes que maximizan, descontando el riesgo y el tiempo, sus beneficios y utilidades; los factores son móviles, las curvas de oferta son elásticas, las influencias institucionales son escasas, de manera que los mercados de productos y factores muestran un amplio grado de competencia (Bustelo, 1999: 116). Estas teorías del desarrollo responden generalmente a dichos enfoques; algunas veces se ha puesto atención al grupo estructuralista y en otras los cambios se han fundamentado en los enfoques neoclásicos. El problema es que las políticas económicas del mundo en desarrollo muchas veces se han basado o se han justificado con estas teorías. Los resultados nos dejan ver que aquellos países que se han distanciado de las recomendaciones hechas por los organismos internacionales sobre política económica tienen mejores resultados que aquellos que las han seguido sin discriminación.

En los años cuarenta, las teorías explicaban el subdesarrollo por la insuficiencia de inversión y ahorro en los países pobres. La transformación estructural se lograría mejorando la tasa de ahorro, permitiendo la entrada de capital extranjero y la intervención del Estado. El comercio internacional era deseable, pero insuficiente para impulsar el desarrollo; por ello se permitía el proteccionismo a las industrias y un déficit moderado en las cuentas externas que se cubría con la inyección de capital extranjero (Ibarra, 2004: 14). A excepción de Myrdal, los pioneros del desarrollo no consideraban el conflicto de intereses entre países desarrollados y subdesarrollados; consideraban que la modernización era siempre positiva, el tradicionalismo siempre negativo, y que los sectores modernos y tradicionales estaban desconectados (Bustelo, 1999: 128). 
En la década de los setenta, se identificó como problema la insuficiencia de cuadros empresariales que no permitían absorber los recursos de exterior ni llevar a cabo los programas del gobierno y de los organismos internacionales. Para solucionarlo se fomentó la formación empresarial, se creó una banca de desarrollo, se exhortaron las inversiones conjuntas en áreas estratégicas de la economía y se fortaleció la capacidad nacional de preparación y evaluación de proyectos; estas propuestas mantenían un enfoque keynesiano (Ibarra, 2004: 14).

Al final de la década de los setenta, cuando terminó el periodo de crecimiento que la economía capitalista había iniciado al terminar la Segunda Guerra Mundial, se presentaron desórdenes en el sistema monetario internacional, disminuyeron los ritmos de crecimiento de capital fijo del sector privado, descendió la tasa media de ganancia empresarial y aumentó la tasa de desempleo (Rueda, 2000).

El nuevo diagnóstico situó el problema en una estructura distorsionada de precios que limitaba la absorción de la mano de obra y generaba tasas subóptimas de expansión del producto. Se condenó la intervención del Estado porque empeoraba la asignación de recursos, propiciaba el uso de técnicas de alta densidad de capital y daba lugar a rentas improductivas acogidas al favor oficial (Ibarra, 2004: 14).

Los países en desarrollo pusieron énfasis en bajar la inflación y crear un clima propicio para el comercio y la inversión de capital extranjero. Entre 1985 y 1989, la inflación mundial se incrementó 16\%; entre 1990-1999 subió hasta 30\% y del 2000 al 2003 bajó a 4\%. En este periodo, el alza promedio de precios declinó de 4 a $2 \%$ en las naciones avanzadas, de 48 a menos de $6 \%$ en las economías en desarrollo y de 186 a 8\% en América Latina. Para lograr esta estabilidad de precios se dio la apertura comercial en forma indiscriminada, se cambiaron las leyes de inversión extranjera, se privatizaron las empresas estatales, se perdió la independencia de los bancos centrales, se instaló una disciplina fiscal extrema que no permitía reaccionar ante ciclos económicos y se retiró la intervención del Estado en la economía, manteniéndose la política de libre mercado, a pesar de que los resultados no respondían a las demandas sociales. El ritmo de desarrollo latinoamericano en más de dos décadas se redujo a la mitad del que alcanzó en los 30 años anteriores y se produjo desempleo, marginación y desajustes en el mercado de trabajo (Ibarra, 2004: 12).

Desde finales de la década de los noventa se ha generado una discusión sobre los efectos de estos ajustes estructurales en América Latina. Ha habido un consenso en comprender la estabilidad macroeconómica más allá del control fiscal, políticas monetarias restrictivas para controlar la inflación, liberalización comercial, liberalización financiera, proce- 
sos de desregulación y privatización. Se ha reconocido la importancia de que existan y se generen instituciones, su financiamiento, el combate a la corrupción y la pobreza, las redes sociales y la gobernanza empresarial (corporate governance) (Dussel, 2004: 3).

Algunas instituciones - como el Banco Interamericano de Desarrollo (BID) y la Comisión Económica para América Latina y el Caribe (CEPAL) - han llamado a replantear la estrategia macroeconómica para los países en vías de desarrollo, mientras que el Fondo Monetario Internacional (FMI) insiste en consolidar y profundizar su estrategia a pesar de que América Latina, evidentemente, no ha recuperado su senda de crecimiento desde la década de los ochenta, a diferencia de gran parte de Asia y especialmente China que no se han sujetado a los cambios en la política económica sugeridos por este organismo (Dussel, 2004: 3).

Las economías del sudeste asiático, especialmente China, se han incorporado al desarrollo sin seguir los lineamientos del Banco Mundial o del Fondo Monetario Internacional. En estos países, el Estado y las élites dominantes se unieron para definir e implementar una política económica que protegió sus intereses, al mismo tiempo que se integraron a los mercados mundiales.

Al comparar los resultados de las reformas económicas implantadas en Latinoamérica con el éxito de las reformas económicas implementadas en Asía, resalta la importancia de la intervención del gobierno en la economía y se ha redescubierto al Estado como agente conductor de la transición hacia las nuevas reglas globales (Ibarra, 2004; World Bank, 1997).

\section{La industria automotriz china, su creación y evolución}

China inició gestiones poco exitosas para producir automóviles desde la década de los treinta. Fue hasta 1956 que fundó la primera fábrica automotriz llamada First Auto Works (FAW), en la provincia de Changchun, con tecnología importada de la Unión Soviética. En 1958, durante el gobierno maoísta, se buscó la autosuficiencia, por lo que los gobiernos locales realizaron inversiones para desarrollar la industria automotriz. En 1964, China tenía 417 plantas que producían camiones, autos, motocicletas y principalmente partes automotrices; para 1976 se tenían 1950 fábricas, pero sólo cuatro producían más de 1000 unidades (Harwit, 1995: 21). Ésta era una industria fragmentada, con altos costos de producción y tecnologías obsoletas. 
A mediados de la década de los sesenta se construyó la Second Auto Works, llamada posteriormente Dong Feng Motor Co. Estos años fueron de lento desarrollo para la industria; en 1970 la producción total fue de 25,100 vehículos, en su mayoría camiones o vehículos utilitarios como las ambulancias (Harwit, 1995: 21). Después de 1976, las autoridades empezaron a promover la especialización y coordinación de las fábricas automotrices creadas en las dos décadas pasadas. Con esto se buscaba la formación del oligopolio para lograr reducir costos, alcanzando economías de escala y, así, mejorar la base tecnológica.

En 1999, la industria automotriz incluía 2,391 empresas entre plantas fabricantes de vehículos y de autopartes, además empleaba 1.8 millones de personas. A pesar de su ineficiencia, la industria dejaba utilidades espectaculares porque los vehículos tenían precios en promedio hasta $33 \%$ más elevados que en el resto del mundo. En 2000, las ventas en la industria alcanzaron 4,600 millones de dólares con utilidades de 2,100 millones, lo que representa el 45.6\% (Owen, 2002: 3).

La importancia que da el gobierno por desarrollar la industria automotriz se observa en el Noveno Plan Quinquenal (1996-2000) en donde se considera industria prioritaria y se realizan proyecciones de crecimiento en la producción y el consumo. En el Décimo Plan Quinquenal (2001-2005) se señala lo siguiente:

[...] se buscará el establecimiento de dos o tres grupos automotrices internacionales y competitivos. Estos grupos tendrán el 70\% del mercado y los servicios y los sistemas estarán de conformidad con las prácticas internacionales. Existirán de 5 a 10 grupos de autopartes con habilidades competitivas primarias y los tres más importantes productores de partes estratégicas tendrán el 70\% del mercado doméstico. Las exportaciones de partes deberán representar el $20 \%$ del total de las ventas. $^{2}$

\footnotetext{
${ }^{2}$ Véase el texto completo en inglés en http://english.peopledaily.com.cn/features/lianghui/zhureport.html
} 


\section{Gráfica 1 \\ Producción de vehículos en China (1986-2004) \\ unidades}

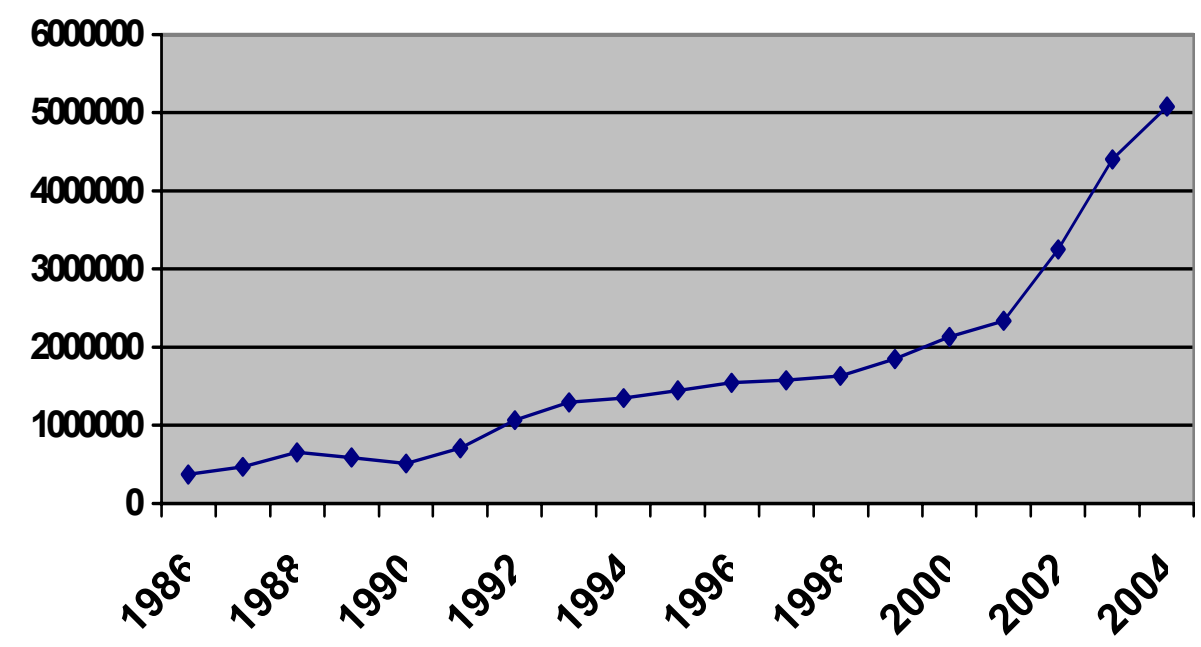

Fuentes: China Automotive Industry Year Book, citado en Wang, 2002;

www.fourin.com; marzo, 2005

Industria Nacional de Autopartes en México. Incluye vehículos de pasajeros, camionetas y camiones

En la gráfica 1 se puede observar la evolución de la producción de vehículos automotores en los últimos veinte años. En China, el consumo estuvo controlado en la segunda mitad del siglo pasado debido a que el automóvil se consideraba un lujo y no se permitía su propiedad privada; fue hasta 1985 cuando se autorizó el consumo individual de camiones, autobuses y barcos, por lo que se observa un crecimiento notable en la producción partir de 1986.

En 1990, el gobierno realizó nuevos ajustes en su estrategia y otorgó prioridad al crecimiento de la producción de automóviles y autobuses. El mercado tuvo varios cambios, la demanda aumentó y hubo un rápido incremento en el consumo individual que se reflejó en un aumento del 50\% de la producción entre 1991 y 1992 pasando de 710 mil a 1.06 millones de unidades. Desde entonces ha habido un incremento constante en la producción con un crecimiento espectacular entre 2001 
y 2003 en que se duplicó la producción pasando de 2.3 a 4.2 millones de vehículos. El crecimiento responde a la demanda del pujante mercado chino y al deseo de las ensambladoras de invertir en un país en el que las utilidades de la industria automotriz son superiores al promedio mundial de la industria.

\section{Cuadro 1}

Ventas de vehículos en China por segmento 2001-2004

\begin{tabular}{|l|c|c|c|c|}
\hline \multicolumn{1}{|c|}{ Ventas } & $\mathbf{2 0 0 1}$ & $\mathbf{2 0 0 2}$ & $\mathbf{2 0 0 3}$ & Mayo-2004 \\
\hline $\begin{array}{l}\text { Autos de } \\
\text { pasajerøs }\end{array}$ & 722,793 & $1,121,846$ & $1,941,342$ & $2,326,492$ \\
\hline Canniones & $\$ 19,500$ & $1,074,392$ & $1,206,959$ & $1,525.91$ \\
\hline Autdouses & 822,399 & $1,064,403$ & $1,181,678$ & $1,218,611$ \\
\hline $\begin{array}{l}\text { Ventas } \\
\text { totales }\end{array}$ & $2,364,692$ & $3,261,141$ & $4,329,979$ & $5,071,011$ \\
\hline
\end{tabular}

Fuente: elaboración propia con información proporcionada por la Industria Nacional de Autopartes, documento de trabajo, y www.fourin.com, "Passenger Car Sales by Maker and Model", marzo, 2005.

En el cuadro 1 se puede observar la distribución de la producción en tres segmentos: autos de pasajeros, camiones y autobuses. El segmento de automóviles es el que tuvo el crecimiento más evidente: $221.87 \%$, pasando de 722793 a 2.3 millones de unidades entre 2001 y 2004 . A continuación, se explican los cambios en la política industrial que van ligados al crecimiento de la industria.

\section{Política económica y desarrollo de la industria automotriz china}

El gobierno chino realizó un esfuerzo para concebir una política completa con el fin de favorecer el desarrollo de una industria nacional. Las leyes de inversión extranjera directa, las políticas de consumo y los altos aranceles a las importaciones le confirieron veinte años a la industria automotriz china para reestructurarse, consolidarse y adquirir capacidades que le ayudarán a competir ante la apertura comercial. 


\subsection{Medidas de protección arancelarias}

Para favorecer la producción local, el gobierno chino impuso numerosos frenos a la importación de vehículos y de autopartes. En 1986, las tarifas de aduana sobre las importaciones de vehículos iban de $180 \%$ para vehículos con motores de menos de tres litros a $220 \%$ para vehículos con motores de más de tres litros; pero la tarifa arancelaria fue objeto de bajas sucesivas como se observa en el cuadro 2 . Uno de los compromisos más importantes que adquirió China al entrar a la Organización Mundial del Comercio (OMC) es la reducción de la tarifa arancelaria, por lo que para el 2006 se espera que baje hasta el 25\%. Aún así la tarifa es muy alta en relación con otras economías en que el arancel es cero.

\section{Cuadro 2}

Tarifas de aduana sobre importaciones de vehículos Porcentaje

\begin{tabular}{|c|c|c|c|c|c|c|c|c|c|c|c|}
\hline Prockicto & $\begin{array}{c}1980 \\
\%\end{array}$ & $\begin{array}{c}1004 \\
\%\end{array}$ & $\begin{array}{c}1990 \\
\%\end{array}$ & $\begin{array}{c}1997 \\
\%\end{array}$ & $\begin{array}{c}2001 \\
\%\end{array}$ & $\begin{array}{c}2 \omega 2 \\
\%\end{array}$ & $\begin{array}{c}2003 \\
\%\end{array}$ & $\begin{array}{c}2004 \\
\%\end{array}$ & $\begin{array}{c}2005 \\
\%\end{array}$ & $\begin{array}{c}2006 \\
\%\end{array}$ & $\begin{array}{c}200 \% \\
\%\end{array}$ \\
\hline $\begin{array}{l}\text { Velúculos } \\
-3 \text { litros }\end{array}$ & 180 & 110 & 100 & 5 & 64 & 438 & 38.2 & 342 & 30 & 28 & 25 \\
\hline $\begin{array}{l}\text { Velúculos } \\
+3 \text { linos }\end{array}$ & 220 & 150 & $\overline{120}$ & 100 & 78 & 50.7 & $\overline{43}$ & 376 & 30 & 28 & $\overline{25}$ \\
\hline
\end{tabular}

Fuente: W Wang y Owen D. Nee Jr, "Automotive Industry in China", China Auto 2002: Shanghai, septiembre 16, 2002.

*Compromisos con la OMC

Las medidas arancelarias manifestaron varias debilidades. En primer lugar, las altas tarifas no impedían el paso de importaciones ilegales atraídas por la enorme demanda que estuvo mucho tiempo comprimida; en 1993 el 19\% de los vehículos en China provenían del contrabando y en 1997 el porcentaje había crecido a 33\% (Wang, 1995: 36). En segundo lugar, la protección aduanera hizo fluctuar el precio de los vehículos fabricados localmente; aprovechando la fuerte demanda, los fabricantes aumentaron los precios y al mismo tiempo vendieron productos de mala calidad; por ejemplo, en el caso de Shanghai VW, el precio de venta del 
modelo Santana en el mercado chino en 1993 era alrededor de 200000 yuans por auto y el costo de producción de alrededor de 85000 yuans: el precio era el doble antes de impuestos. En esta industria protegida, el beneficio era superior a la media de la industria. De hecho, el índice de rentabilidad en 1995 era tres veces más elevado que el conjunto del sector industrial (Wang, 2002: 36).

\subsection{Medidas de protección no arancelaria}

Las cuotas y licencias a la importación, la tasa de integración nacional y las restricciones a la comercialización de productos fueron factores limitantes a la importación. Para conseguir las licencias de importación se estableció un procedimiento confuso: los organismos autorizados para expedir las licencias no estuvieron claramente identificados.

Para incrementar la competitividad de los proveedores locales, una nueva ley de joint-venture fue promulgada en marzo de 2001, la cual permitió a los fabricantes aplicar un abastecimiento internacional, sin obligación de un porcentaje local de partes y componentes comprados en China. Esta nueva etapa de desarrollo acentuó la competitividad de los proveedores después de la construcción de un tejido industrial relativamente completo. Exponer a los proveedores chinos a una competencia internacional los obligó a acelerar la innovación tecnológica para responder a la exigencia de calidad de componentes en el nivel internacional (Gao, 2004).

Otros reglamentos que funcionaban como barrera no arancelaria eran los controles en las divisas extranjeras, así como el derecho de importación y de comercialización de vehículos reservada a la sociedad china; solamente cuatro puertos en China podían importar automóviles: Dalian, Tianjin, Shanghai y Huangpu (Owen, 2002: 5). Gracias a esta clase de proteccionismo pudieron proliferar pequeñas fábricas de autos artesanales porque las economías de escala no eran una condición necesaria para la competencia. La mayoría de estas empresas se establecían y después hacían una solicitud al gobierno central para obtener aprobaciones. A pesar de que sus vehículos no tenían licencia de venta, podían venderse regionalmente protegidos por el gobierno local (Wang, 2002: 37). 


\subsection{El cambio en la política de consumo de vehículos particulares}

El desarrollo de la industria automotriz estuvo frenado por el control del consumo. El primer reglamento que autorizó el consumo individual fue promulgado en 1984; éste se refería a la compra de vehículos, tractores y barcos para los agricultores. El consumo de vehículos particulares se desregularizó en 1990. Los impuestos y los derechos en el nivel del gobierno central y regional también constituyeron serias barreras para la compra. No fue sino a principios del tercer milenio que el consumo de vehículos particulares permitió a la industria automotriz desarrollarse.

Los impuestos y derechos encarecían los costos de uso de un vehículo; el conjunto de impuestos y de derechos nacionales y regionales representaba del 30 al 50\% del precio del auto. Si se aumentaban los costos por derechos de matriculación (entre 15 y $50 \%$ del precio del vehículo), los costos de compra se elevan entre $145 \%$ y $200 \%$ del precio de un auto nuevo. Las autoridades chinas tuvieron que reflexionar sobre el sistema de impuestos y de derechos para poder incentivar el consumo de vehículos. Por ello a partir de 1999 muchas provincias suprimieron una parte de los impuestos y derechos regionales; en total, 238 derechos regionales se suprimieron en 26 provincias y municipios (Wang, 2002: 421).

Actualmente, los precios de los vehículos en China están teniendo una reducción importante. De agosto de 2003 a julio de 2004, 76 modelos fueron vendidos, de ésos 63 tuvieron una reducción en sus precios, pasando de un precio promedio de 21,148 a 19,425 dólares, lo que representa un descuento promedio de $8.5 \%$. El segmento de vehículos de menos de 1.4 litros fue el que mayor descuento registró con $12.1 \%$. Esta estrategia de las empresas fabricantes de automóviles se dio como respuesta a las políticas de financiamiento que se han vuelto cada vez más restrictivas y han puesto a muchos consumidores potenciales fuera del mercado (Fourin, 2005: 5).

\subsection{La inversión extranjera directa y la política industrial del sector automotriz}

Desde la década de los noventa, China se ha convertido en un importante receptor de inversión extranjera directa que sobrepasa a cualquier otro país en desarrollo. La creación de zonas especiales, donde se autorizaba la entrada de capital extranjero y las importaciones libres de aranceles, fue una de las reformas más importantes y en pocos años se convirtió en un poderoso factor de crecimiento económico (Rueda y González, 2002: 174). No obstante que la inversión extranjera 
directa (IED) global anual cayó de 1,388 billones a 560 billones entre 2000 y 2003, ésta mantuvo su crecimiento en China al pasar de 40 billones en 2000 a 53 billones en 2003 y a 60 billones en 2004 (Business Custom Wire, 2005: 1).

\section{Gráfica 2}

\section{Flujos de inversión extranjera directa a China}

Millones de dólares

Fuente: Lee Michael Y. (2003: 44)

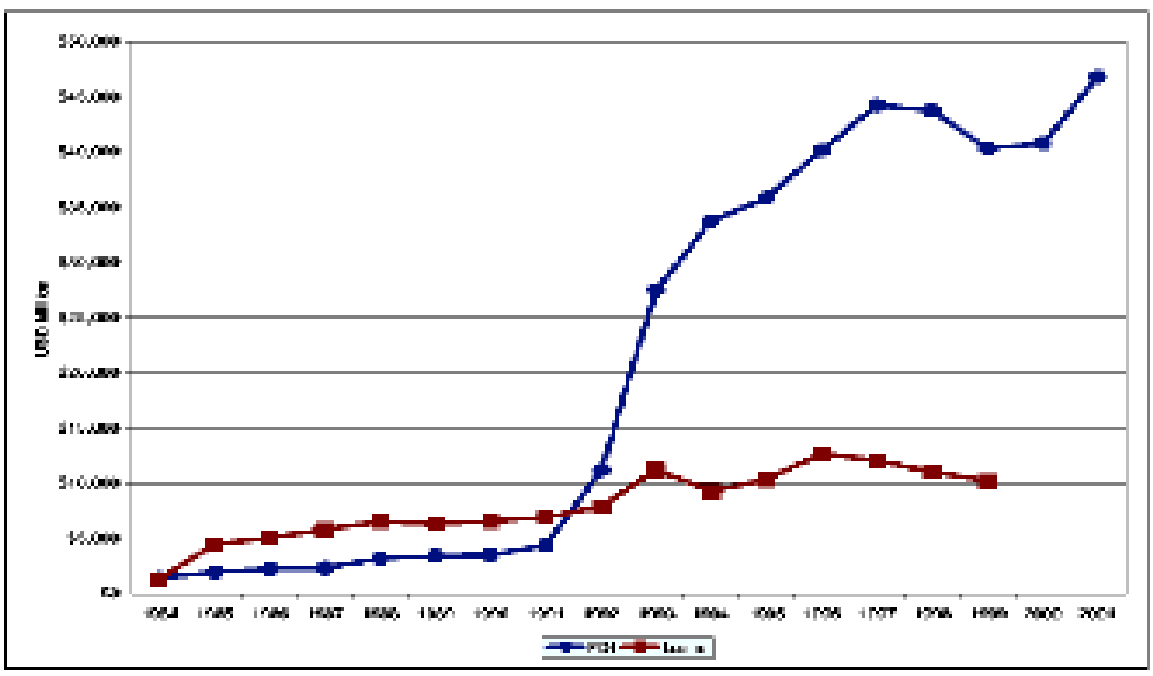

La teoría neoclásica sugiere que la IED es un motor de crecimiento para el país a donde llegan porque puede mejorar la formación del capital bruto y del empleo, puede promover la manufactura y sus exportaciones, puede aumentar su conocimiento administrativo y know how, puede propiciar el acceso a redes de producción internacional y utilizar marcas establecidas; también puede resultar en transferencia de tecnología y tener efectos positivos sobre la creación de empleo. Por otro lado, se pueden señalar algunos efectos negativos porque inhiben el desarrollo de capacidades tecnológicas y administrativas locales, pagan bajos impuestos por los precios de transferencia que adoptan entre la matriz y la filial o por los productos listos para ensamblarse, pueden influir en la política gubernamental en forma desfavorable al exigir protección y trato preferencial, exenciones de impuestos, tie- 
rra y servicios baratos (Holgin, 2001: 681). A continuación se presentan algunos indicadores de los resultados de la participación de la inversión extranjera en la economía china.

\section{Cuadro 3}

Indicadores de participación de la inversión extranjera directa en China

\begin{tabular}{|c|c|c|c|}
\hline & 1991 & 1995 & 1998 \\
\hline $\mathrm{IED}$ c oum porc entaje de la inversión bruta & $390 \%$ & $15.10 \%$ & $15.20 \%$ \\
\hline $\begin{array}{l}\text { Exportaciones de las empresas con IED } \\
\text { enmillones de dolares }\end{array}$ & $12.1 \mathrm{mrl}$ & $46.9 \mathrm{mc}$ & $88.6 \mathrm{~mm}$ \\
\hline $\begin{array}{l}\text { Partic pacion en las exportaciones de las } \\
\text { empresas con IED }\end{array}$ & $17.00 \%$ & $3130 \%$ & $44.10 \%$ \\
\hline $\begin{array}{l}\text { Partic pación en elprochuto inchustrial } \\
\text { de las empre as con IED }\end{array}$ & $5.00 \%$ & $11.70 \%$ & $14.90 \%$ \\
\hline No . de emple ados enmillbnes de personas & 48 & 16 & 18 \\
\hline $\begin{array}{l}\text { Imprue stos pagados (\% del total de } \\
\text { immpuestos) }\end{array}$ & $4.10 \%$ & $10.00 \%$ & $13.20 \%$ \\
\hline
\end{tabular}

Fuente: Honglin Zhang, "How does foreing direct investment affect economic growth in China", Economics of transition, vol. 9, 3, 2001.

En el cuadro 3 se puede observar que la IED ha tenido efectos positivos en la economía china: como parte de la inversión bruta pasó de $5 \%$ a $14.9 \%$ en siete años; ayudó a reducir la presión que tiene el gobierno de crear empleos generando 13.2 millones de ellos; asimismo, incrementó su participación con 9 puntos en el total de impuestos recolectados.

Por otro lado, la IED también ha ayudado a transformar la estructura de propiedad en China: en 1992 las empresas propiedad del Estado eran responsables del 48\% del producto interno bruto (PIB); las empresas colectivas, del 38\%; y las privadas, incluyendo a las de capital extranjero, que era mínimo, del 13\%. Seis años después la participación de las empresas propiedad del estado disminuyó 10 puntos porcentuales, pasando a $28.2 \%$; las colectivas se mantuvieron con $38 \%$; las privadas incrementaron sus participación 17.9\%; y las de capital extranjero participa- 
ron con 14.9\% (Honglin, 2001: 683). Además, ha jugado un rol importante al transformar las empresas del estado al introducir prácticas administrativas como métodos de contabilidad, administración de riesgo y sistemas de administración de la producción. La integración de la economía mundial va en aumento y las empresas extranjeras han ayudado a promover sus exportaciones a través de sus redes mundiales; la participación de estas empresas en las exportaciones pasó de $17.0 \%$ a $44.1 \%$ entre 1991 y 1998 (ver cuadro 3).

De igual forma, también hubo algunos efectos negativos en la economía, pues las empresas con IED se unieron a empresas locales para buscar protección y alzar barreras de entradas a productos internacionales. Además, la transferencia de tecnología ha sido limitada en comparación con el nivel esperado, al contemplar el estatus de país en desarrollo y a sus capacidades tecnológicas. Sin embargo, dado el tamaño del mercado, existen muchas oportunidades para aumentar la transferencia de tecnología a través de Joint Venture (Young y Lan, 1996: 669).

\subsubsection{La regulación de inversión extranjera directa y su relación con la in- dustria automotriz}

China estableció desde 1979 un cuadro jurídico complejo sobre la inversión extranjera directa, fomentando la inversión en los sectores conocidos como "favorables" (principalmente los sectores de fabricación orientados a la exportación y en los sectores tecnológicos). En lo que concierne a la industria automotriz, el objetivo fue hacer de esta industria un pilar de la economía.

La ley de inversión extranjera directa definió tres categorías para clasificar a los sectores: de fomento, limitados y prohibidos. La industria automotriz era un sector de fomento y se limitó solamente en la creación de empresas ensambladoras. Debido a que los efectos de la IED en el sector automotriz no tuvieron completamente el efecto deseado (por ejemplo, el reglamento para limitar el ensamblado de vehículos no funcionó eficazmente y se crearon más fábricas de automóviles); en 2001 se definió que únicamente la empresa extranjera que quisiese exportar la totalidad de sus productos se clasificaría en la categoría de fomentado y todas las otras inversiones estarían sujetas a revisión.

Las inversiones en la industria automotriz tenían que ser aprobadas por una agencia oficial central o provincial, dependiendo de la naturaleza y el tamaño de la 
inversión. El proyecto de ensamblaje de vehículos completos, los componentes claves - como el motor, los frenos ABS, bolsas de aire- y todas las inversiones superiores a 30 millones de dólares dependían exclusivamente de los niveles centrales. Con la entrada de China a la Organización Mundial del Comercio (OMC), esta regla cambió, por lo que en 2005 las autorizaciones serán necesarias a partir de 150 millones de dólares, reduciendo así los trámites para las inversiones de las empresas fabricantes de automóviles. Además, la política de la industria automotriz de 2004 señala restricciones a nuevos entrantes: no se autoriza la creación de nuevas plantas y los permisos existentes no se pueden vender o traspasar a empresas fuera del sector (Owen, 2002: 8).

La participación del capital extranjero en una JV de vehículos ensamblados es obligatoriamente minoritaria (o igual). Esta medida puede ser impuesta a la parte extranjera para la producción de ciertos equipos considerados como estratégicos (frenos ABS, motores de inyección, bolsas de aire); sin embargo, en el sector de componentes los inversionistas extranjeros pueden tener el control total de las acciones y de las filiales.

El control de los chinos sobre la JV se efectúa por la participación mayoritaria en el capital de las empresas. Si la autoridad suprime esta medida, las empresas corren el riesgo de ser eliminadas en el sector automotriz, como ha sucedido en otros países, por lo que mantener un porcentaje mayoritario de capital local es económica y políticamente crucial para el gobierno chino. El reglamento actual sobre la condición de la participación de capitales en las JV (capital extranjero igual o menor al 50\%) puede garantizar que los grupos chinos conserven la mitad del mercado. Esta regla influye sobre la tecnología que se transfiere a las JV. Actualmente, se considera que la tecnología que transfieren las transnacionales está adelantada dos años a la industria china y 20 años atrasada con relación a la tecnología de la empresa transnacional. Se prevé que mientras se mantenga la restricción sobre el porcentaje de inversión extranjero en la empresa, la tecnología no fluirá libremente. Además, existen serios problemas con las leyes de propiedad intelectual que inhiben la transferencia de tecnología. Durante el año 2004 se acusó a Chery, empresa de capital chino, de haber copiado su vehículo QQ del Chevrolet Spark $^{3}$ (http://thecarconection.com).

${ }^{3}$ Las autoridades señalaron que no había suficientes elementos que probaran que el vehículo había sido copiado. Estos acontecimientos nos dan idea de las capacidades tecnológicas chinas y la aplicación de la incipiente ley de propiedad industrial. 


\subsubsection{La Política Industrial del Sector Automotor}

La Política Industrial del Sector Automotor (PISA), promulgada en 1994, nunca constituyó una ley o regulación en sentido formal; sin embargo, fue exitosa porque la inversión extranjera en el sector automotriz siempre estuvo bajo el escrutinio del gobierno central debido a que se requería la aprobación de la Comisión Estatal de Planeación y Desarrollo o de la Comisión Estatal de Economía y Comercio (Owen, 2002: 3).

La PISA se operó en el cuadro de una política articulada alrededor de dos grandes ejes: la incitación y la reglamentación; asimismo se invitó a las empresas automotrices a usar las inversiones extranjeras para desarrollar la industria automotriz china.

La política estableció prioridades para desarrollar productos para la industria doméstica: a) componentes y partes automotrices, b) autobuses de pasajeros, c) camiones y motocicletas y d) facilidades para procesos. El Estado creó un préstamo-preferencia de capital foráneo para camiones de pasajeros, camiones de carga, motocicletas y manufactura de partes. Se estableció un sistema de autentificación de los productos antes de ser vendido, importado o usado para controlarlos. Una vez autentificado el vehículo, se registraba en un catálogo nacional usado por la oficina de seguridad pública en el momento de otorgar la licencia del vehículo. Asimismo, para aprobar los proyectos de inversión extranjera directa era necesario tener socios con las siguientes características: a) patentes propias de productos y marcas, b) tecnología para desarrollo de productos y manufactura, c) organización de ventas internacional independiente y d) capacidades financieras adecuadas (Owen, 2002: 5-9).

Las Joint Venture estaban obligadas a tener su propia investigación y desarrollo, contar con productos que cubriesen los estándares técnicos de calidad mundial, dar estatus preferencial para partes domésticas y componentes; por su parte, el socio chino debe poseer por lo menos el 50\% de las acciones (Owen, 2002: 5-9).

La nueva PISA, publicada en junio de 2004, tiene como actor principal al consumidor de vehículos particulares e incluye reglas para su protección y la del ambiente. En éstas se prevén y estimulan el crédito para el consumo de vehículos y de servicios como los seguros, talleres automotrices y estacionamientos en las ciudades. 


\subsubsection{Flujos de inversión extranjera directa en el sector automotriz}

La inversión extranjera directa en la industria automotriz china inicia en 1983 con la Joint Venture Beijin Jeep; para 1996 la industria había acumulado 15.4 billones (Wang, 2002: 37); en 1998 alcanzó 20.3 billones de dólares repartidos de la siguiente manera: 11.04 billones en ensambladoras de vehículos completos, 9.0 billones en autopartes y 321 millones en otros vehículos automotores; posteriormente, entre 2001 y 2003 se invirtieron 6.6 billones (www.fdi.gov.cn).

Hong Kong y otros países de Asia fueron los primeros inversionistas de la industria automotriz china, sobre todo en motocicletas y vehículos especiales como ambulancias; su tecnología era intensiva en mano de obra y más fácil de transferir. La inversión europea y de los EUA se enfocó en vehículos para pasajeros y han logrado una posición dominante aprovechando la política de gobierno (Wuang, 1999: 5).

\section{Cuadro 4}

Indicadores de desempeño de la industria automotriz en 1997

\begin{tabular}{|l|c|c|c|}
\hline & Joint Ventioes & $\begin{array}{c}\text { Enquesas del } \\
\text { Estado }\end{array}$ & Otras \\
\hline $\begin{array}{l}\text { Productividad } \\
1000 \text { yuand mo. } \\
\text { enqleados }\end{array}$ & 564.1 & 104.1 & 279 \\
\hline $\begin{array}{l}\text { Utilidades por } \\
\text { enpleado }\end{array}$ & 28.2 & 1 & 29.1 \\
\hline $\begin{array}{l}\text { Retoun sobre la } \\
\text { inverión }\end{array}$ & 4.2 & 0.5 & 12.54 \\
\hline $\begin{array}{l}\text { Retoun sore } \\
\text { ventic }\end{array}$ & 556 & 0.98 & 9.68 \\
\hline
\end{tabular}

Fuente: Wuang, 1999: 6

En 1997, los indicadores financieros mostraban que las utilidades por empleado, el retorno sobre la inversión y ventas, las JV se desempeñaron mejor que las empresas propiedad del Estado, pero no tuvieron mejor desempeño 
que otras particulares. Sin embargo, la productividad en las armadoras europeas, americanas y japonesas era cuatro veces más alta que el promedio de la industria.

\section{La estructura de la industria y la inversión extranjera directa}

El gobierno ha realizado serios esfuerzos por formar el oligopolio, y aunque aún están registradas 128 empresas, 15 grupos chinos fueron responsables del $84 \%$ de la producción en 2004; también encontramos 23 fabricantes de camiones y autobuses, 18 fabricantes de camiones y 24 fabricantes de autobuses.

\section{Cuadro 5 \\ Ventas de vehículos de los grupos automotrices chinos en unidades}

\begin{tabular}{|l|c|c|c|}
\hline \multicolumn{1}{|c|}{ Grupo } & $\mathbf{2 0 0 3}$ & $\mathbf{2 0 0 4}$ & \% de cambio \\
\hline FAW & & & \\
\hline Dongfeng Motor & 903,777 & $1,013,300$ & 12.1 \\
\hline SAIC & 471,594 & 503,308 & $6.70 \%$ \\
\hline Chang'an & 782,036 & 848,542 & 8.5 \\
\hline Norinco & 410,745 & 504,805 & $22.90 \%$ \\
\hline Beijing & 3,219 & 6,537 & 103.1 \\
\hline Guangzhou Automobile & 336,657 & 530,993 & 57.7 \\
\hline Yuejin Motors & 122,608 & 209,551 & 70.9 \\
\hline $\begin{array}{l}\text { Brilliance Automotive } \\
\text { Holdings }\end{array}$ & 100,280 & 95,275 & -5 \\
\hline Geely Group & 118,869 & 99,572 & -16.2 \\
\hline China Aviation & 81,252 & 105,879 & 30.3 \\
\hline Total de los grupos chinos & 324,666 & 314,342 & -3.2 \\
\hline Ventas totales de la industria & $\mathbf{4 , 3 9 0 , 6 1 9}$ & $\mathbf{5 , 0 7 1 , 0 6 1 *}$ & $\mathbf{1 5 . 5}$ \\
\hline
\end{tabular}

Fuente: Elaboración propia con información de Fourin, "Ventas por grupos automotrices chinos, 2004", marzo, 2005. Incluye autos, camiones y autobuses. 
Los grupos chinos tienen estructuras muy complejas porque se formaron de varias empresas que pertenecían a diferentes estados. El gobierno reconoce como grupo a un conjunto de empresas en donde una de ellas tiene propiedad o control sobre tres empresas o más; por ejemplo, FAW se divide en tres niveles:

a) En el primer nivel tiene 39 fábricas que controlan directamente, tres subsidiarias, tres centros de investigación y escuelas; además, tiene 581 compañías de servicio dedicadas a producir componentes y otros servicios.

b) En el segundo nivel se encuentran nueve empresas sobre las que se tiene control; entre éstas se encuentra la JV FAW-Volkswagen, que es la mayor productora de automóviles en China. También se encuentra Qingdao Automobile Works y la Dalian Diesel Engine plant; estas tres empresas han sido centros de producción para vehículos de pasajeros, camiones diesel y motores diesel, respectivamente, y son importantes participantes de la estrategia de diversificación de FAW.

c) El tercer nivel se compone de 136 empresas, universidades y centros de investigación en los que FAW no tiene capital, pero que producen autopartes o se han unido a FAW para tener fácil acceso como proveedores (Marukawa, 1995: 344).

Los fabricantes de automóviles mundiales han ingresado al mercado automotriz desde 1983 y paulatinamente han ido creciendo mediante la asociación con estos grupos chinos. La primer JV establecida en China fue Beijing Jeep, relacionada con Chrysler, a finales de 1983. Durante los años noventa se incrementó, en promedio, una cada año; sin embargo, a partir del año 2001 se registró un crecimiento sin precedentes al registrarse ocho asociaciones, impulsadas por la entrada de China a la OMC.

En el cuadro 6 se observa que el líder en la producción de automóviles particulares durante la última década ha sido Volkswagen y sus dos JV con los principales grupos chinos FAW y SAIC abarcando $27 \%$ del mercado; el año pasado el vehículo más vendido en China fue el Jetta — que tuvo una producción de 153,916 unidades abarcando el $6.2 \%$ del mercado-y el Santana - que tuvo una producción de 132, 713 unidades abarcando el 5.3\% del mercado. Es importante señalar que a pesar de que se mantienen como líderes, Shanghai VW perdió 5 puntos porcentuales en la participación de mercado y FAW-VW apenas creció $0.7 \%$ entre 2003 y 2004 . 


\section{Cuadro 6}

Participación de mercado de las empresas productoras de automóviles particulares 2004

\begin{tabular}{|c|c|c|c|c|}
\hline Joint Ventures & $\begin{array}{l}\text { Socio } \\
\text { chino } \\
\end{array}$ & $\begin{array}{c}\text { Socio } \\
\text { extranjero } \\
\end{array}$ & $\begin{array}{c}\% \text { de } \\
\text { participación de } \\
\text { mercado } 2003\end{array}$ & $\begin{array}{c}\% \text { de } \\
\text { participación de } \\
\text { mercado } 2004\end{array}$ \\
\hline $\begin{array}{l}\text { 1. First } \\
\text { Automitve } \\
\text { Works-VW }\end{array}$ & FAW & VW & 19.6 & 14.39 \\
\hline $\begin{array}{l}\text { 2. Shanghai } \\
\text { Volkswagen }\end{array}$ & $\begin{array}{l}\text { Shanghai } \\
\text { Automotive } \\
\text { Group (SAIC) }\end{array}$ & VW & 14.7 & 12.9 \\
\hline $\begin{array}{l}\text { 3. Shanghai } \\
\text { General } \\
\text { Motors }\end{array}$ & SAIC & GM & 9.96 & 10.83 \\
\hline $\begin{array}{l}\text { 4. Guangzhou } \\
\text { Honda }\end{array}$ & $\begin{array}{l}\text { Guangzhou } \\
\text { Automobile } \\
\text { Industry }\end{array}$ & Honda & 5.7 & 8.6 \\
\hline $\begin{array}{l}\text { 5. Beijin } \\
\text { Hyundai }\end{array}$ & Beijin Motors & Hyundai & 2.5 & 6.19 \\
\hline $\begin{array}{l}\text { 6. Chang' an } \\
\text { Susuky }\end{array}$ & Chang & Susuky & 4.95 & 4.7 \\
\hline $\begin{array}{l}\text { 7. Dongfeng } \\
\text { Motors }\end{array}$ & Dongfeng & Peugeot-Citroes & 5.1 & 3.83 \\
\hline 8. FAW-Toyota & $\overline{F A W}$ & Toyota & 2.3 & $3 ., 5$ \\
\hline $\begin{array}{l}\text { 9. Chang'an } \\
\text { Ford }\end{array}$ & Chang'an & Ford & 0.008 & 2 \\
\hline 10. Beijin jeep & Beijín Motors & Chrysler & 0.9 & 1.28 \\
\hline 11. FAW-Honda & FAW & Honda & 0.0001 & 0.006 \\
\hline 12. Otros & & & 33.6 & 31.52 \\
\hline
\end{tabular}

Fuente: elaboración propia con información de Fourin, "Ventas de vehículo por fabricante", marzo, 2005

En tercer lugar se encuentra General Motors, que a partir del 1997 hizo su aparición y ha creado siete $\mathrm{JV}$, lo que ha permitido obtener un crecimiento y participación de mercado del 9\%; su producto más importantes es el Biuick Regal del cual vendió 72,908 unidades el año pasado abarcando el 2.9\% del mercado. 
Guangzhou Honda ha tenido un crecimiento importante, puesto que su participación en el mercado cambió de 5.7 a $8.6 \%$ entre 2003 y 2004; su modelo Accord vendió 105,385 unidades, que representan el 4.2\% del mercado. Beijin Hyundai fue la JV que más incrementó su participación en el mercado pasando de 2.5 a $6.19 \%$ en el periodo mencionado; su principal producto es el modelo Santa Fe. Finalmente, las JV de Peugeot, Toyota, Ford, participan con menos del 5\% del mercado, respectivamente. A continuación se presentan las características más importante de las cuatro JV más importantes.

\section{Shanghai Volkswagen Automotive Company}

La JV se estableció en 1985; en 2004 firmó un acuerdo de ampliación de operaciones hasta el 2030. Volkswagen y SAIC incrementaron la inversión en 181 millones con lo que llegó a un total de 942 mil millones de dólares y se situó como la JV con mayor inversión en el sector automotriz. (www.volkswagen.com.cn) Su producción se inició con el modelo Santana, único vehículo en China que alcanzó ventas por 215,547 unidades en 2003; también fabrica Passat, Polo y Golf. Esta empresa ha estado nominada por ocho años consecutivos como una de las 500 empresas más grandes; durante el periodo 1995-2000 se convirtió en el primer fabricante en el sector automotriz que cubría más del $50 \%$ del mercado; además, desde su inicio ha fabricado más de 1.38 millones de vehículos.

\section{First Automotive Works- Volkswagen Automotive Company (FAW- VW)}

Esta sociedad se creó en 1989; su principal modelo es el Jetta del cual fabrica 150,000 unidades por año. En 1996, introdujo una versión moderna del Audi con una producción de 30,000 unidades en 2001; inició la producción del Bora y al siguiente año la de Audi 6. Desde 1994 produce motores y cajas de velocidad.

\section{Shanghai General Motors Company (SGM)}

Shanghai General Motors Company Limited (Shanghai GM) se estableció en junio de 1997; produce vehículos, motores y transmisiones automotrices. Desde su fundación ha invertido más de 2000 millones de dólares y ha sido líder en la introducción de nuevos productos a partir de 1999, incluyendo los Buicks New Century, GLX, GL, GL8 y G. En 2001 comenzó a exportar el GL8 a Filipinas; asimismo, empezó a vender el modelo Buick SVR; en 2003, el Buick 
Excelence. Su estrategia va dirigida al mercado de automóviles de lujo (www.gm.com/china).

\section{Guangzhou Honda Automobile}

Honda entró al mercado chino en 1982 produciendo motocicletas mediante un acuerdo de colaboración técnica con una compañía local; ha registrado un crecimiento constante hasta establecer un centro de investigación y desarrollo en 2003. La JV se estableció en 1998 con una inversión inicial de 133 millones de dólares por un periodo de 30 años. En 1999 comenzó la producción del modelo Accord en Guangzhou y fue el primer fabricante de vehículos automotores en establecer una red independiente de servicios y ventas en China (www.honda.com/worldwide).

\section{Reflexiones finales}

Las leyes e instituciones son fuerzas que determinan la estructura de la industria. En este trabajo se explicó cómo la férrea intervención del Estado, las leyes e instituciones inducen el surgimiento de una industria automotriz con centenas de fabricantes y miles de proveedores: la fragmentación de la industria, el sistema paralelo en el que se fabrican vehículos del campo, la evolución de medidas proteccionistas y la lenta adopción de políticas de apertura de la industria automotriz permitieron la creación de capacidades de producción importantes que dejan a China en una posición competitiva importante.

La IED fue estrictamente supervisada por el Estado; la regla de inversión (igual o menor del $50 \%$ para capital extranjero) ha ayudado a mantener el control del mercado en manos chinas, pero la presión para quitar esta regla irá en aumento conforme el país se integre más al comercio mundial y sus importaciones y exportaciones dependan de los países miembros de la OMC. A partir de ahora observaremos una lucha constante entre las empresas fabricantes de automóviles que buscan ganar espacio en la comercialización, financiamiento, aseguramiento y servicio de los vehículos que producen e importan a China y el gobierno y los grupos chinos que buscarán adquirir capacidades tecnológicas y administrativas que les permitan competir con los grupos multinacionales en un futuro cercano.

El objetivo del gobierno chino con respecto a crear dos o tres grandes grupos automotrices que pudieran competir con las empresas fabricantes de automóviles 
en el nivel mundial se ha alcanzado de forma parcial. Desde 2004 existen cinco grupos chinos que cumplen las expectativas de los planes de desarrollo: First Auto Works (FAW), Shanghai Automotive Industy Corporation (SAIC), Beijin Motors, Chang'an y Dongfeng Auto. El mercado de camiones y autobuses está dominado por los grupos chinos; de hecho, en la producción de camiones de menos de 6 toneladas, Dongfeng y Faw ocupan el segundo y tercer lugares de producción en el nivel mundial; sin embargo, el mercado de automóviles que es el de mayor crecimiento en China está dominado por las Joint Venture que estos grupos tienen con VW, GM, Honda, Hyundai y Susuki.

La inversión extranjera directa en forma de JV participa de manera importante en la producción de automóviles particulares y se están formando tejidos intricados con la creación de muchas otras JV que evolucionan de las actuales. Hay claros beneficios a partir de la inversión extranjera como el incrementó en la participación de las exportaciones, la participación en la creación de capital bruto y las contribuciones fiscales. También se ha creado empleo aunque la productividad de las JV y de las empresas particulares de capital local han llevado a disminuir el número de empleos en la industria.

El estudio de la industria automotriz china, su crecimiento y desarrollo, nos permite reflexionar cómo el gobierno chino y sus empresarios logran a través del control de la inversión extranjera y del esfuerzo propio desarrollar capacidades que ahora les permiten participar en una industria que sirve a su mercado, les da empleo y crea valor que se transforma en utilidades, impuestos y riqueza para sus ciudadanos.

En México, durante el periodo de sustitución de importaciones, se pretendió que la inversión extranjera participara con conocimientos y tecnología mientras los empresarios mexicanos invertían recursos y desarrollaban capacidades; sin embargo, los empresarios no desarrollaron las capacidades necesarias y nunca se logró diseñar y fabricar vehículos y componentes en México. De hecho, los decretos automotrices y la forma en que se aplicaron propiciaron la desaparición de empresas de capital local, en primera instancia. En la actualidad, el capital local tiene una participación mínima en la industria automotriz: las empresas armadoras y los proveedores de primer nivel son empresas transnacionales y el capital local participa únicamente como proveedor de segundo y tercer niveles.

Actualmente, la industria automotriz en México busca la forma de bajar costos en los vehículos y algunos ya empiezan a incluir hasta $30 \%$ de partes y componentes 
manufacturados en China; ésta es una amenaza que las empresas de autopartes en México deben enfrentar. Asimismo, es claro que la estrategia de las ensambladoras prevalecerá y serán éstas las que decidan el contenido de partes chinas en los vehículos ensamblados en México, basándose en los costos y la calidad necesarias.

La industria de autopartes en China representa una seria amenaza para la industria automotriz en México, por lo que es necesario establecer acciones para superar las debilidades. China se ha convertido en el segundo socio comercial de los Estados Unidos solamente después de Canadá y ha desplazado a México al tercer lugar a partir de 2003. La venta de autopartes chinas a los Estados Unidos se ha incrementado notablemente, así que existen probabilidades de perder participación en el mercado principal si no se reacciona adecuadamente.

Por otro lado, también existen oportunidades de cooperación; pues hay empresas mexicanas que han seguido a las transnacionales y ahora exportan algunos materiales como piel para asientos e interiores de vehículos de lujo de General Motors. La industria automotriz china se perfila como una de las más importantes del mundo; seguir su evolución se vuelve imprescindible para todos aquellos que tengan probabilidades de competir con ella.

\section{Bibliografía}

ARCE, Randall y Eduardo Gitli, "El ingreso de China a la OMC y su impacto sobre los países de la cuenca del Caribe", revista de la CEPAL núm. 74, 2001.

BUSTELO, Pablo, Teorías contemporáneas del desarrollo económico, Síntesis, España, 1999, p. 303.

FOURIN-a, "China 2010 automobile market forecast: 7 million vehicles and global number2", www.fourin.com, march, 2004.

FOURIN-b, "Monthly statistics overview", www.fourin.com, febrero, 2, 2005.

FOURIN-c, "Passanger car sales by market and model", www.fourin.com, marzo, 2005 
FOURIN-d "Pasanger car price cuts: average price drops $8.5 \%$ in one year to 148 thousand RMB", www.fourin.com, sept, 2004

GAO, Paul, "Shaping the future of China's auto industry", The McKinsey Quartely, 2004, núm. 3. EUA.

GAO, Paul, "A tune-up for China's auto industry", The McKinsey Quartely, 2002, no. 28, EUA.

HARWIT, Eric,"China's Automobile Industry Policies, Problems and Prospects", East Book Gate, New York, 1995, p.208.

HOFFE J. Lane K., Miller V, "Branding cars in China", The McKinsey Quartely,2003, núm. 3.

HONGLIN, Kevin, "How does foreign direct investment affect economic growth in China", Economics of Transition, 2001, vol. 9 (3) 679-693.

HUBLER, Jerome and Pierre-Xavier Meschi, "European direct investment in China and sino-french Joint Ventures", Asia Pacific Business Review,2001, vol 7, no. 3 spring, pp 157,180.

IBARRA, David, "Los vericuetos del orden internacional: la importación de reformas", Economiaunam, enero-abril, 2004, pp., 7-18.

LARDY, Nicholas, China in the World Economy, Institute for International Economics, Washington, EUA, 1998.

LEE, Michael Y., Changing Dynamicas of the Chinise Automotive Indutry: The Impact of Foreign Investment, Technology Transfer and WTO Membership, tesis de Maestria, Massachusetts Institute of Technology, june, 2003.

PINGYAO, Lai, "Foreign direct investment in China: recent trends and patterns", China \&World Economy, 2002. no.2, pp 25-32.

RUEDA PEIRO, Isabel y María L. González Marín, "Crecimiento y cambios socio económicos en China: 1978-2000", Problemas del Desarrollo, Instituto de Investigaciones Económicas, UNAM, núm. 128, México, 2002. 
UNCTAD, "Informe sobre el Comercio y el desarrollo 2002"; Capítulo V, La Adhesión de China a la OMC y Naciones Unidas,2002, http://www.unctad.org/sp/ docs/tdr2002_sp.pdf

WANG HUA, Restructuration de L'Industrie Automobile Chinoise, quelle Trajectorie dans la Mondialisation, tesis doctoral, 2002, Universidad Pierre Mendes, Francia.

WANG HUA y Richett Xavier, "Foreign direct investment in the chinese automotive industry", China's Perspectives, núm. 38. 2001.

WAYNE W.J. Xing, "China's Automobile Market- The Last and Largest Untapped Market in the World", 1998.

WTO, "Evaluación del comercio mundial en 2002 y perspectivas para 2003", World Trade Organization, 2002, http://wto.org/spanish/res_s/statis_s/ its03_general_overview

YOUNG, Stephen y Ping Lan, "Technology transfer to China through foreign direct investment", Regional Sutides, 1996, vol. 31.7 p, 669-679.

\section{Paginas electrónicas:}

www.beijin-jeep.com

www.cacauto.com

www.chinacars.com

Www.chinaonline.com

www.china.org.com

www.cei.gob.cn

www.faw.com

www.fourin.com

www.ford.com

www.fdi.gov.cn

www.fiat.com

www.gm.com

www.geely.com.cn

www.honda.com

www.hyuandai-motor.com 